\title{
Übersicht
}

Obere Extremität 2021 · 16:94-107 https://doi.org/10.1007/s11678-021-00646-9 Eingegangen: 16. Dezember 2020

Angenommen: 8. April 2021

Online publiziert:22. Mai 2021

c c Der/die Autor(en) 2021
Raphael Blunier ${ }^{1}$ (D) - Marvin Lucas Frommer ${ }^{1}$ Matthias Zumstein ${ }^{1,2}$. Michael Schär ${ }^{1}$

'Abteilung für Orthopädische Chirurgie und Traumatologie, Inselspital, Universität Bern, Bern, Schweiz

${ }^{2}$ Abteilung für Schulter-, Ellbogen und Sportsorthopädie, Sonnenhof Orthopädisches Center, Bern, Schweiz

\section{Plättchenkonzentrate in der Behandlung von Rotatorenmanschettenläsionen}

\section{Narrativer Review}

Trotz stetiger Verbesserungen bei der Refixationstechnik von Rotatorenmanschettenrupturen (RMR) ist die Rerupturrate nach wie vor hoch. Aus diesem Grund wird versucht, die Manschette zusätzlich mit Biologika wie z. B. Plättchenkonzentraten zu augmentieren. Neben der einfachen Gewinnung und Herstellung gibt es für diese autolog gewonnenen Konzentrate relativ tiefe regulatorische Hürden. Dass diese Konzentrate nur Minuten nach der Blutentnahme und Zentrifugation wieder appliziert werden können, ist ein weiterer Vorteil. Dieser Artikel befasst sich mit der Frage, inwieweit die verschiedenen Plättchenkonzentrate die Manschettenheilung nach Refixation positiv beeinflussen.

\section{Hintergrund}

Die RMR treten häufig auf und deren Inzidenz nimmt im Alter zu [1]. Bei einer stetig wachsenden alten Population, welche zudem immer aktiver wird, wird auch in Zukunft die Menge an RMR-Rekonstruktionen weiter zunehmen. Zwar zeigen viele Studien in einer hohen Prozentzahl der Fälle ein gutes klinisches Outcome [2-5] nach Rotatorenmanschetten- (RM-)Rekonstruktion. Trotz stetiger Verbesserung der Refixationstechnik zeigt sich aber trotzdem nach wie vor eine durchschnittliche Rerupturrate von $26,6 \% 24$ Monate

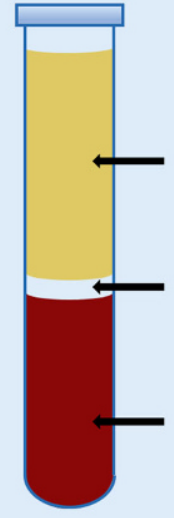

a
Plasma

(55\% des Probenvolumens)

Buffy Coat

Leukozyten und Plättchen ( $<1 \%$ des Probenvolumens)

Erythrozyten

(45\% des Probenvolumens)

Abb. $1 \Delta$ a Bei der Zentrifugation mit hoher Zentrifugationsgeschwindigkeit entstehen bei L-PRP (,leucocyte and platelet-rich plasma") drei Schichten: Die obere Schicht besteht aus purem Plasma, die mittlere Schicht enthält das „buffy coat", das die Plättchen und die Leukozyten enthält. Die untere dunkelrote Schicht besteht aus Erythrozyten. b Gewinnung von „platelet-rich fibrin“ (PRF)
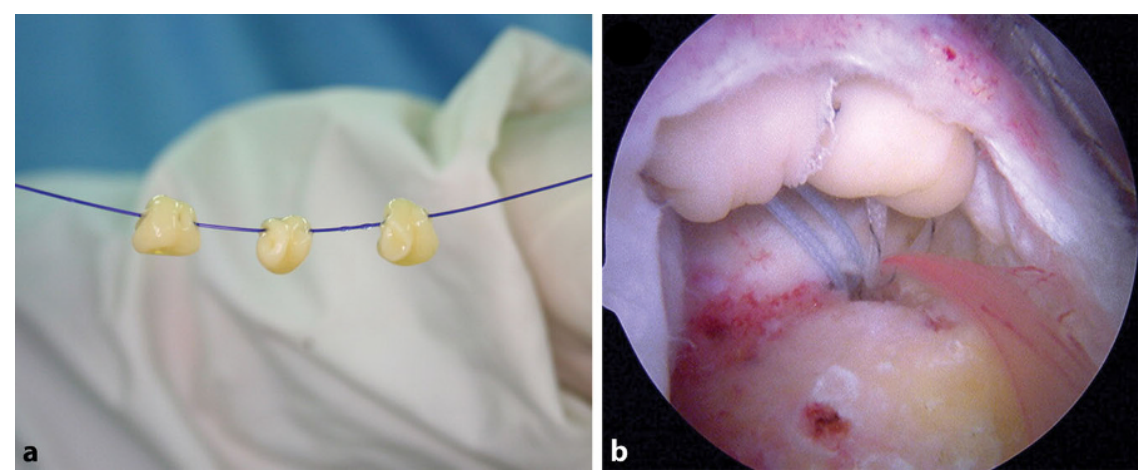

Abb. $2 \Delta$ a Vor der arthroskopischen Applikation werden die drei P-PRP-Gels („pure platelet-rich plasma") mittels eines No. 1 PDSII miteinander verbunden. b Die so entstandene Kordel wird dann zwischen den Footprint und die Sehne geklemmt. (Aus [20] mit freundl. Genehmigung von AJSM) 
Tab. 1 Auf dem Markt erhältliche zugelassene Plättchenkonzentrate und deren Konzentration mit Einteilung in die jeweilige Plättchenkonzentratgruppe

\begin{tabular}{|c|c|c|c|}
\hline L-PRP & P-PRP & L-PRF & P-PRF \\
\hline $\begin{array}{l}\text { GPS III System } \\
\text { Zimmer Biomet } \\
\text { IN, USA } \\
\text { Plättchen: 5-9,3× konz. } \\
\text { Leukozyten: } 5 \times \text { konz. } \\
\text { Harvest SmartPreP } 2 \\
\text { und } 3 \\
\text { Terumo BCT Inc. } \\
\text { Terumo Group, } \\
\text { Lakewood, CO, USA } \\
\text { Plättchen: 3-4× konz. } \\
\text { Magellan } \\
\text { Arteriocyte } \\
\text { Hopkinton, MA, USA } \\
\text { Plättchen 3-5× konz. } \\
\text { Pure PRP SP } \\
\text { Emcyte } \\
\text { Fort Myers, FL, USA } \\
\text { Plättchen: } 7 \times \text { konz. } \\
\text { Orthopras } \\
\text { Proteal } \\
\text { Barcelona, Spanien } \\
\text { Plättchen: } 2,8 \times \text { konz. } \\
\text { Angel System } \\
\text { Arthrex } \\
\text { Naples, FL, USA } \\
\text { Plättchen: 4,1-18×konz. } \\
\text { Tubex } \\
\text { Moohan Enterprise } \\
\text { Hoedeok-gil, Gyeonggi } \\
\text { do, Südkorea }\end{array}$ & $\begin{array}{l}\text { Nahita PRP } \\
\text { Nahita, Navarra, } \\
\text { Spanien } \\
\text { Plättchen: 2,2× konz. } \\
\text { ACP } \\
\text { Arthrex, FL, USA } \\
\text { Plättchen: 2-3× konz. } \\
\text { COBE Spectra LRS } \\
\text { Turbo } \\
\text { Terumo BCT Inc. } \\
\text { Terumo Group, } \\
\text { Lakewood, CO, USA } \\
\text { Plättchen:6,1× konz. } \\
\text { A-PRP RegenKit } \\
\text { Regen Lab } \\
\text { Le Mont-Sur, } \\
\text { Lausanne, } \\
\text { Schweiz } \\
\text { Plättchen 1,6× konz. } \\
\text { PRGF-Endoret } \\
\text { BTI Technology } \\
\text { Institute, } \\
\text { Álava, Spanien } \\
\text { Plättchen: } 2,6 \times \text { konz. } \\
\text { Angel System } \\
\text { Arthrex } \\
\text { Naples, FL, USA } \\
\text { Plättchen: 4,1× bis } \\
\text { 18× konz. } \\
\text { Orthopras } \\
\text { Proteal } \\
\text { Barcelona, Spanien } \\
\text { Plättchen: } 2,8 \times \text { konz. } \\
\text { MyCells } \\
\text { ProTech, Kaylight } \\
\text { Cop., Orange, CA, USA } \\
\text { Plättchen: 4-5× konz. } \\
\text { Prosys PRP } \\
\text { Prodizen } \\
\text { Seongnam-si, Gyeonggi } \\
\text { do, Südkorea } \\
\text { Plättchen: 8,04× konz. } \\
\text { Leukozyten: }\end{array}$ & $\begin{array}{l}\text { Intra-Spin } \\
\text { L-PRF } \\
\text { Intra-Lock } \\
\text { International } \\
\text { Birmingham, } \\
\text { AL, USA } \\
\text { Choukrons } \\
\text { L-PRF } \\
\text { A-PRF } \\
\text { Process for } \\
\text { PRF } \\
\text { Nizza, Frank- } \\
\text { reich }\end{array}$ & $\begin{array}{l}\text { Fibrinet } \\
\text { Royal Biologics } \\
\text { Hackensack, NJ, USA } \\
\text { Vivostat PRF } \\
\text { Vivostat A/S, } \\
\text { Medicon Valley, } \\
\text { Dänemark } \\
\text { Plättchen: } \\
\text { 2,6-3,9× konz. } \\
\text { Cascade Autologous } \\
\text { Platelet System } \\
\text { Musculoskeletal } \\
\text { Transplant Foundation } \\
\text { Edison, NJ, USA } \\
\text { Plättchen: } 3 \times \text { konz. } \\
\text { PRGF-Endoret } \\
\text { BTI Technology } \\
\text { Institute, } \\
\text { Álava, Spanien } \\
\text { Plättchen: } 2,6 \times \text { konz. }\end{array}$ \\
\hline
\end{tabular}

postoperativ über die gesamte RMR-Rekonstruktionspopulation, wobei massive und große Defekte $(>3 \mathrm{~cm})$ 4,06-mal häufiger rerupturieren als kleine und mittlere Läsionen $(0-3 \mathrm{~cm})$ [6]. Auch das Patientenalter beeinflusst die Rerupturrate. Bei einer Alterszunahme von 10 Jahren steigt die Rerupturrate um den Faktor 1,67. Weitere Faktoren, die die Rerupturrate negativ beeinflussen, sind Einzelreihenrekonstruktionen, Rekonstruktionen mit modifizierter Mason-
Allen-Nahttechnik sowie längere postoperative Immobilisation [6].

Wegen dieser nach wie vor hohen Rerupturrate wird heute versucht, die Manschettenheilung zusätzlich mit biologischen Mitteln $\mathrm{zu}$ verbessern. In diese Kategorie fällt die Augmentation mittels Plättchenkonzentraten, von welchen man sich u. a. eine Modulation der Entzündungsreaktion, verbesserte Durchblutung, erhöhte Tenozytenzahl, vermehrte Einwanderung von Stammzellen und verbesserte Analgesie und somit insgesamt einer Verbesserung und Beschleunigung der Heilung und weniger Rerupturen erhofft $[7,8]$. Neben der einfachen Gewinnung und Herstellung der Plättchenkonzentrate gibt es für diese Produkte relativ tiefe regulatorische Hürden, da es sich um autolog gewonnene Konzentrate handelt, die unmittelbar nach der Blutentnahme und Zentrifugation appliziert werden können.

Während in In-vitro-Studien und Tierversuchen nach Applikation von Plättchenkonzentraten $\mathrm{z}$. T. vielversprechende Ergebnisse gezeigt werden konnten mit verbesserter Heilungsrate [9-11], zeigen klinische RMR-Rekonstruktionsstudien nach zusätzlicher Applikation von Plättchenkonzentraten bislang kein klares einheitliches und/oder klar positives Resultat bezüglich deren Nutzen. Diese sehr variablen Resultate in klinischen Studien sind u.a. auch damit zu erklären, dass Plättchenkonzentrate kein einheitliches Produkt darstellen. 2014 zeigte eine Cochrane-Metaanalyse [12], bei der alle Plättchenkonzentratstudien vereinigt wurden, keinen Benefit bezüglich einer zusätzlichen Applikation von Plättchenkonzentraten. 2019 zeigte dann eine darauf folgende CochraneMetaanalyse einen Benefit von Plättchenkonzentraten, wenn appliziert nach einer RM-Rekonstruktion [13]. Solche Studien sind gefährlich, implizieren diese doch, dass potenziell jedes Plättchenkonzentrat einen Nutzen zeigt. Dies ist aber nicht der Fall, wie wir in diesem Reviewartikel zeigen möchten. Aus diesem Grund werden wir die Plättchenkonzentrate in 4 Untergruppen unterteilen.

\section{Einteilung der Plättchen- konzentrate}

Plättchenkonzentrate werden aus dem venösen Vollblut des Patienten durch Plasmapherese hergestellt, welches kurz vor oder während dem operativen Eingriff dem Patienten entnommen wird. Sie sind somit ein autologes Produkt. Dabei werden verschiedene Schemata mit einer unterschiedlichen Dauer, Anzahl der Durchgänge und Zentrifugationsgeschwindigkeiten verwendet. Bei manchen Produkten ist zusätzlich das Hinzufügen eines Antikoagulans oder 
eines anderen Zusatzstoffes notwendig, um die spezifischen Eigenschaften des Produkts $\mathrm{zu}$ erreichen. Ein mit Antikoagulantien (z.B. Citrat) versetztes Teströhrchen beinhaltet nach der Zentrifugation in der obersten Schicht leukozytenarmes Blutplasma, den Leukozytenfilm in der mittleren Schicht, auch „buffy coat" genannt und Erythrozyten in der untersten Schicht. Das Produkt bleibt durch das Antikoagulans flüssig. Je nach Zentrifugationsprozess befinden sich die Plättchen im „buffy coat“ (hohe Zentrifugationsgeschwindigkeit) oder im Plasma (tiefe Zentrifugationsgeschwindigkeit). Im Gegensatz dazu verbinden sich in einem Teströhrchen ohne Antikoagulans einige der Blutbestandteile durch die Zentrifugation zu einer fibrinösen Masse. Das entstandene Fibrin kann dann als solides Produkt ganz anders als das flüssige Produkt verwendet werden. Zum Beispiel kann es während der Operation in den Defekt eingenäht werden.

Die verschiedenen Herstellungsmöglichkeiten bilden die Grundlage einer sinnvollen Unterteilung in Untergruppen. Die folgenden Ausführungen basieren auf der Einteilung von Dohan Ehrenfest et al. [14]. Sie unterteilen die verschiedenen Plättchenkonzentrate nach derem Anteil an Leukozyten in reine Plättchenkonzentrate (ohne Leukozyten) sowie leukozytenreiche Konzentrate (- Tab. 1). Jede dieser zwei Gruppen wird zudem weiter in eine Plasma- und eine Fibrinuntergruppe unterteilt. Während die Konsistenz in den zwei Plasmagruppen (L-PRP [„leucocyte and platelet-rich plasma“] und P-PRP [„pure platelet-rich plasma“]) eher der von Flüssigkeit entspricht, zeigen die zwei Fibringruppen (L-PRF [„leucocyte and platelet-rich fibrin“] und P-PRF ["pure platelet-rich fibrin"]) eine stabile Fibrinstruktur von soliderer Konsistenz. Ein solches Fibrin kann bei der Operation sogar eingenäht werden.

Das P-PRP beschreibt die „wässrigen “ Plättchenkonzentrate mit wenig Leukozyten und plättchenreichem Plasma (- Abb. 1a und 2). L-PRP sind die „wässrigen“ Konzentrate mit hohem Leukozyten- und Plättchenanteil (• Abb. 3). Produkte mit einem Fibrinanteil werden

Obere Extremität 2021 · 16:94-107 https://doi.org/10.1007/s11678-021-00646-9

(c) Der/die Autor(en) 2021

R. Blunier · M. L. Frommer · M. Zumstein · M. Schär

Plättchenkonzentrate in der Behandlung von Rotatorenmanschettenläsionen. Narrativer Review

\section{Zusammenfassung}

Hintergrund. Trotz stetiger Verbesserungen bei der Refixationstechnik von Rotatorenmanschettenrupturen (RMR) ist die Rerupturrate mit $26,6 \%$ nach wie vor hoch. Aus diesem Grund wird heute deshalb versucht, die Manschettenheilung zusätzlich mit biologischen Mitteln zu augmentieren. In diese Kategorie fällt die Augmentation mittels Plättchenkonzentraten. Neben der einfachen Gewinnung und Herstellung gibt es für diese autolog gewonnenen Konzentrate relativ tiefe regulatorische Hürden. Dass diese Konzentrate nur Minuten nach der Blutentnahme und Zentrifugation wieder appliziert werden können, ist ein weiterer Vorteil. Der Begriff Plättchenkonzentrate stellt eine große inhomogene Gruppe von verschiedenen Konzentraten mit unterschiedlichen Eigenschaften dar. Bedingt durch unterschiedliche Herstellungsprozesse weist nicht jedes Plättchenkonzentrat die gleichen Eigenschaften auf.

Fragestellung. Ist die biologische Augmentation mit Plättchenkonzentraten bei Rotatorenmanschettenläsionen mit einem verbesserten Outcome verbunden? Wie unterscheiden sich die gängigen Plättchenkonzentratprodukte in ihrem klinischen und radiologischen Resultat?

Material und Methoden. Die Suche fand via PubMed mit Einschluss von 26/289 Studien von 2007 bis 2020 statt.

Ergebnisse und Diskussion. Die Evaluation der heute verfügbaren Studien zeigt, dass nur P-PRP („pure platelet-rich plasma“) zur biologischen Augmentation von RMR-Refixationen empfohlen werden kann. Hier zeigte sich eine signifikant tiefere Rerupturrate in den P-PRP-Gruppen. Aber auch in dieser Plättchenuntergruppe sind die Resultate heterogen. Bei der konservativen Therapie von Partialrupturen und Tendinopathien der Manschette zeigt sich kein Benefit nach biologischer Augmentation mittels Plättchenkonzentrate.

Schlüsselwörter

Plättchenreiches Fibrin · Plättchenreiches Plasma · PRP · PRF · Biologische Augmentation

\section{Platelet concentrates for the treatment of rotator cuff tears. Narrative review}

\section{Abstract}

Background. Despite improving surgical techniques for rotator cuff repair, the retear rate is still high at $26 \%$. That is the reason why nowadays we try to improve healing with biological augmentation, i.e., with platelet concentrates. Beside the easy production of those autologous concentrates, the regulator barriers are quite low. The timely use of those products immediately after the blood collection is a further advantage of platelet concentrates. The term platelet concentrates represents a large inhomogeneous group of different concentrates with various properties. Due to different manufacturing processes, not every platelet concentrate product has the same properties.

Objectives. Can the use of platelet concentrates in rotator cuff tears improve outcome? How different is the outcome between the different products?
Materials and methods. Through PubMed we assessed 26 studies from 2007-2020. Results and conclusion. The evaluation of the studies which are currently available shows that only P-PRP (leucocyte poor platelet rich plasma) can be suggested for the augmentation of rotator cuff repair. In some studies, significant lower retear rates for the P-PRP group were reported. However, the results are heterogeneous. This puts the benefit of P-PRP into perspective. Platelet concentrates did not improve healing or the clinical outcome after conservative therapies of partial ruptures and tendinopathies.

\section{Keywords}

Platelet-rich fibrin · Platelet-rich plasma · PRP . $\mathrm{PRF} \cdot$ Biological augmentation 


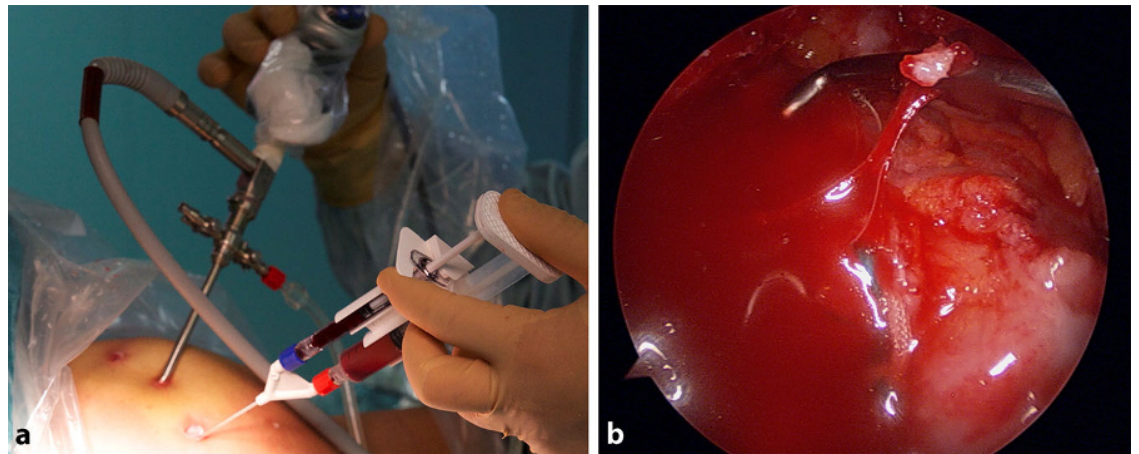

Abb. $3 \Delta$ a Mittels speziellem Applikator wird das L-PRP (leukozytenreiches plättchenreiches Plasma) durch das Arthroskopieportal auf die Rotatorenmanschette gespritzt. b Das applizierte P-PRP gerinnt auf der Manschette. (Aus [18], mit freundl. Genehmigung von Elsevier)

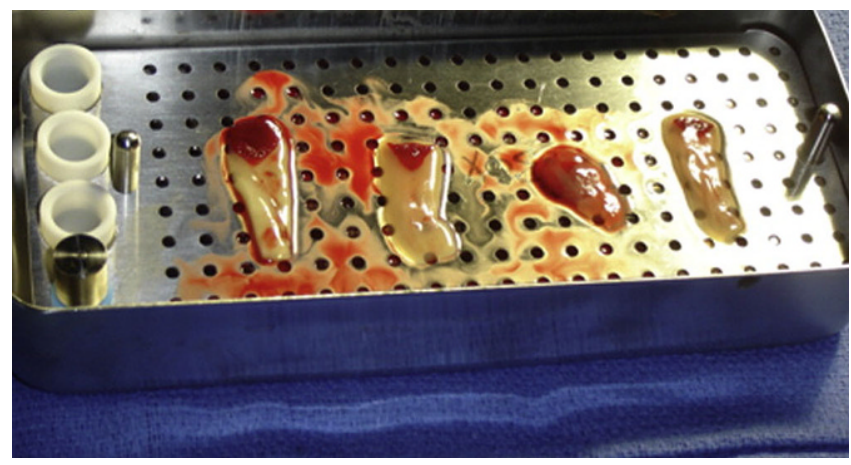

Abb. $5 \Delta$ Auf der Abbildung sind 4 L-PRF („leucocyte and platelet-rich fibrin“) zu sehen. Bedingt durch die relativ stabile Fibrinstruktur können diese Fibrine sogar eingenäht werden. Mit diesen L-PRF-Koageln kann die Manschette nun augmentiert werden. (Aus [33], mit freundl. Genehmigung von Elsevier)

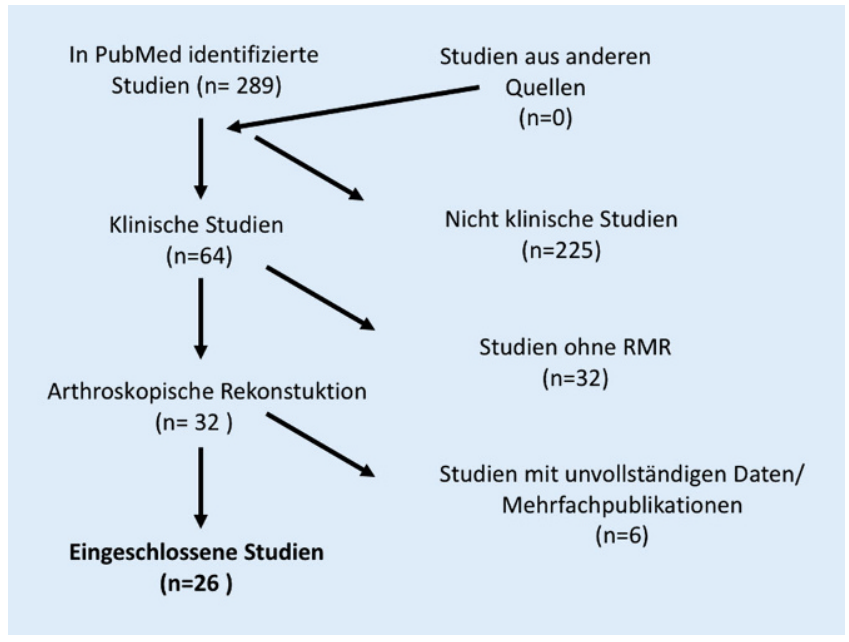

Abb. $6<$ Flussdiagramm zur Übersicht der analysierten Studien (RMR Rotatorenmanschettenruptur)

analog in das leukozytenarme plättchenreiche Fibrin (P-PRF; - Abb. 4) und in das leukozytenreiche plättchenreiche Fibrin (L-PRF; - Abb. 1b) unterteilt (• Abb. 5).

Mit dieser Einteilung erhofft man sich eine bessere Unterteilung der vielen Plättchenkonzentrate auf dem Markt und zu-

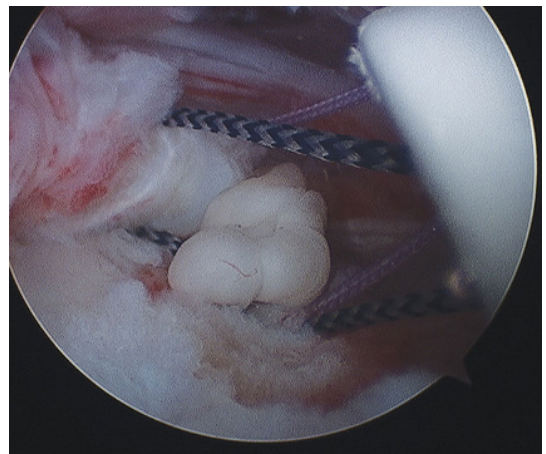

Abb. 4 \ Nach der Herstellung der P-PRF-Koagel („pure platelet-rich fibrin“) werden diese mittels PDS-Fäden in Sandwich-Technik zwischen Footprints und Sehne fixiert. (Aus [39] mit freundl. Genehmigung von AJSM)

\section{Material und Methoden}

Hierzu suchten wir (RB, LF und MOS) mit der PubMed-Suchfunktion klinische Studien über Plättchenkonzentratanwendung bei RMR und Partialrupturen (Suchbegriffe: Platelet-rich + cuff) im Zeitraum von 2007 bis 2020 (• Abb. 6).

Folgende Ausschlusskriterien wurden auf die so 289 gefunden Studien angewandt:

- nicht-klinische Studien,

- Studien, bei denen keine RMR durchgeführt wurde (z.B. konservative Therapie mit Plättchenkonzentrataugmentation),

- Studien mit nur einem Untersuchungsarm,

- Studien mit unvollständigen Daten,

- Mehrfachpublikationen.

In einem zweiten Schritt wurden Studien evaluiert, bei denen mittels Plättchenkonzentraten Partialrupturen und Tendinopathien der RM augmentiert wurden.

Die Unterteilung der Plättchenkonzentratprodukte vollzogen wir nach Ehrenfest et al. [14] in pures plättchenreiches Plasma (P-PRP), leukozyten- und plättchenreiches Plasma (L-PRP), pures plättchenreiches Fibrin (P-PRF) und leukozyten- und plättchenreiches Fibrin (LPRF).

Wir schlossen 26 Studien (Level I-III) mit insgesamt 1567 Probanden ein: 5 Studien zu L-PRP, 10 Studien zu P-PRP, 3 Studien zu L-PRF und $8 \mathrm{zu}$ P-PRF. Zwar wird in jeder Gruppe der gleiche Plättchenkonzentrattyp appliziert, die Studi- 


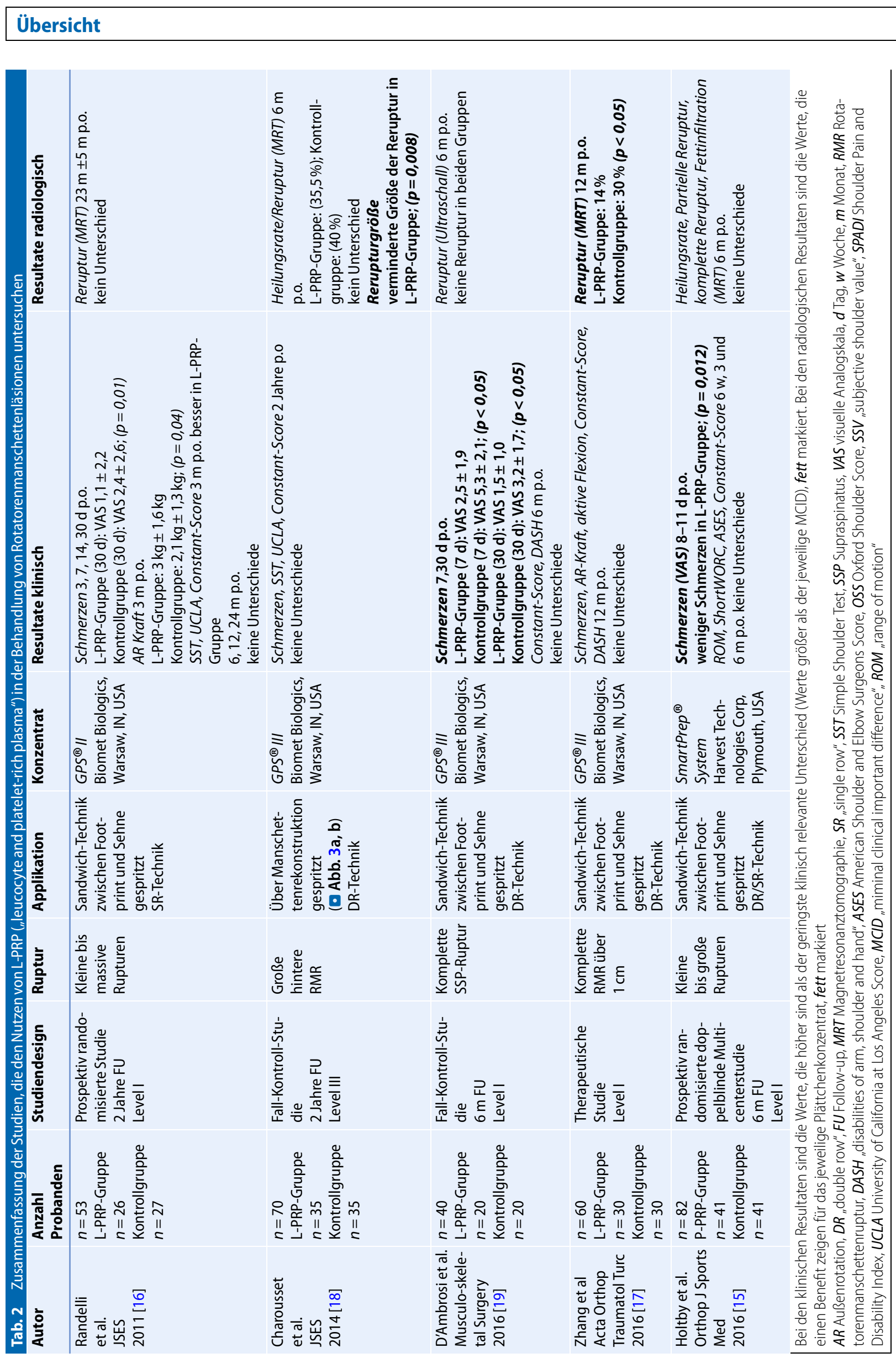


Tab. 3 Metaanalyse von Hurley et al. aus dem Jahr 2019 [13] aller Studien, bei denen der Benefit von L-PRP („leucocyte and platelet-rich plasma“) auf die Rotatorenmanschettenheilung untersucht wurde

\begin{tabular}{|c|c|c|c|c|}
\hline Outcome & $\begin{array}{l}\text { Anzahl Patienten } \\
\text { L-PRP/Kontrolle }\end{array}$ & $\begin{array}{l}\text { Outcome } \\
\text { L-PRP/Kontrolle }\end{array}$ & Signifikanz & $\begin{array}{l}\text { Gewinner im } \\
\text { klinischen Alltag }\end{array}$ \\
\hline \multicolumn{5}{|l|}{$L-P R P$} \\
\hline Sehnenheilungsrate & $72 / 73$ & $30,5 \% / 40,7 \%$ & $p=0,36$ & Keiner \\
\hline Constant-Score & $72 / 73$ & $86,0 / 76,6$ & $p=0,11$ & Keiner \\
\hline
\end{tabular}

en sind jedoch inhomogen bezüglich refixierter Sehnen, Rupturgröße, Volumen und Konzentration des Plättchenkonzentrats und deren Applikationsort, was die Evaluation nicht ganz einfach macht.

\section{Nutzen der Plättchen- konzentrate nach RM- Rekonstruktion}

\section{Nutzen von L-PRP}

Hierzu analysierten wir 5 Studien: 2 prospektive RCT („randomized controlled trial“, $[15,16])$, eine therapeutische Studie [17] und 2 Fall-Kontroll-Studien [18, 19] der vergangenen 9 Jahre (• Tab. 2). Ob die Augmentation bei Manschettenrefixationen mittels L-PRP einen Benefit zeigt, kann mit der bestehenden Evidenz zum heutigen Zeitpunkt nicht konklusiv beurteilt werden. In 3 Studien fanden sich verminderte Schmerzen im ersten Monat postoperativ in den jeweiligen L-PRPGruppen im Vergleich zur Kontrollgruppe ohne L-PRP-Applikation [15, 16, 19]. Zudem zeigte sich eine verbesserte AuBenrotationskraft 3 Monate postoperativ in der L-PRP-Gruppe [16]. Der geringe Benefit von verminderten Schmerzen im ersten postoperativen Monat und einer verbesserten Außenrotation 3 Monate p.o. muss kritisch gegenüber dem doch größeren Aufwand gesehen werden. Alle anderen Studien zeigen keinen klinischen Benefit nach Applikation von L-PRP [17-19].

Auch bezüglich der Rerupturrate zeigt sich nach Applikation von L-PRP kein klarer Trend. Zwar zeigten Zhang et al. ([17]; Level-I-Studie) 12 Monate postoperativ eine Rerupturrate von nur $14 \%$ in der L-PRP-Gruppe im Vergleich zu $40 \%$ in der Kontrollgruppe. Alle ande- ren 4 Studien (3-mal Level I und einmal Level III) fanden jedoch keine Unterschiede bezüglich der Rerupturrate [15, 16, 18].

Auch nach Poolen der Datensätze (Metaanalyse von Hurley et al. [13]) zeigte sich kein Benefit von L-PRP (- Tab. 3).

\section{Nutzen von P-PRP}

Die P-PRP-Produkte sind die am meisten verbreiteten und auch durch Studien am besten dokumentierten Plättchenkonzentrate. Wir schlossen 10 Studien ein: eine prospektive Kohortenstudie [20], eine prospektive randomisierte doppelblinde Studie [21], 7 RCT [22-28] und eine konsekutive Serie [29] - mitunter die größte untersuchte Studie mit 120 Probanden (•Tab. 4).

Auch bezüglich P-PRP zeigt sich keine eindeutige Evidenz bezüglich dem Nutzen zur Augmentation von RMR-Refixationen, wenn man die heute verfügbaren Studien analysiert. Während sich eine verbesserte Heilungsrate und verminderte Rerupturrate in 3 Level-I-Studien fand $[22,23,26]$, zeigten die restlichen 6 von 10 Studien (3 Level-I-, 2 LevelII- und 1 Level-III-Studien), die die Rerupturrate untersuchten keinen Unterschied bezüglich dieser Parameter [20, 24, 25, 27-29]. Auch bezüglich dem klinischen Outcome sind die Resultate der 10 Studien sehr heterogen. Nur vereinzelt zeigen sich klinisch relevante Unterschiede [30-32], z. B. Schmerzen einen Monat p.o. [26], Schmerzen 16 Monate p.o. [29], dem Constant-Score und Shoulder Pain and Disability Index (SPADI) 3 Monate p.o. [20]. Diesen 5 klinisch relevanten Unterschieden stehen aber in den 10 verfügbaren Studien über 54 gemessene
Outcome-Parameter, die nicht relevante Unterschiede zeigten, gegenüber.

Erst nach Poolen der Daten zeigt sich sowohl eine statistisch signifikant verbesserte Sehnenheilungsrate sowie weniger Schmerzen nach 30 Tagen [13]. Im Weiteren zeigt die P-PRP-Gruppe zwar statistisch signifikant bessere Outcome-Scores (Constant-Score und University of California at Los Angeles Score [UCLA]), aber diese Unterschiede sind klinisch nicht relevant (Werte unter dem MCID [ „miminal clinical important difference“]; - Abb. 7; [13]).

Das P-PRP ist die einzige Untergruppe, die zur Augmentation nach RM-Rekonstruktionen empfohlen werden kann, dies aber nur bedingt, denn auch hier sind die Resultate über die verfügbaren Studien sehr heterogen.

\section{Nutzen von L-PRF}

Bei den leukozytenreichen Fibrinkonzentraten untersuchten wir 3 prospektiv randomisierte Studien ([33-35]; - Tab. 5).

Zumstein et al. [33, 34] zeigten eine verbesserte Vaskularisation nach 6 Wochen in der L-PRF-Gruppe, was einen positiven Effekt auf die Heilung haben kann. Ansonsten fand sich aber in keiner der drei Studien ein positiver Effekt nach Applikation von L-PRF. Diese Plättchenkonzentratgruppe kann also für die Augmentation der RMR-Rekonstruktion nicht empfohlen werden.

\section{Nutzen von P-PRF}

Wir untersuchten 2 doppelblinde RCT [36, 37], 4 RCT [38-41], eine Kohortenstudie[42] und eine Fall-Kontroll-Studie ([43]; - Tab. 6). Bei der Augmentation mit P-PRF sind in der Studie von Barber et al. [43] signifikant verminderte Rerupturraten von $30 \%$ für P-PRF beschrieben (Kontrollgruppe 60\%). Andere fanden aber signifikant höhere Rerupturraten verglichen mit der Kontrollgruppe [38, 39]. Rodeo et al. [39] mussten ihre Studie sogar abbrechen und zeigten ein 5,8-mal höheres Risiko für eine Reruptur in der P-PRF-Gruppe 12 Wochen postoperativ [38, 39]. Bergeson et al. [42] fanden ähnliche Resultate mit Rerupturen in 


Übersicht
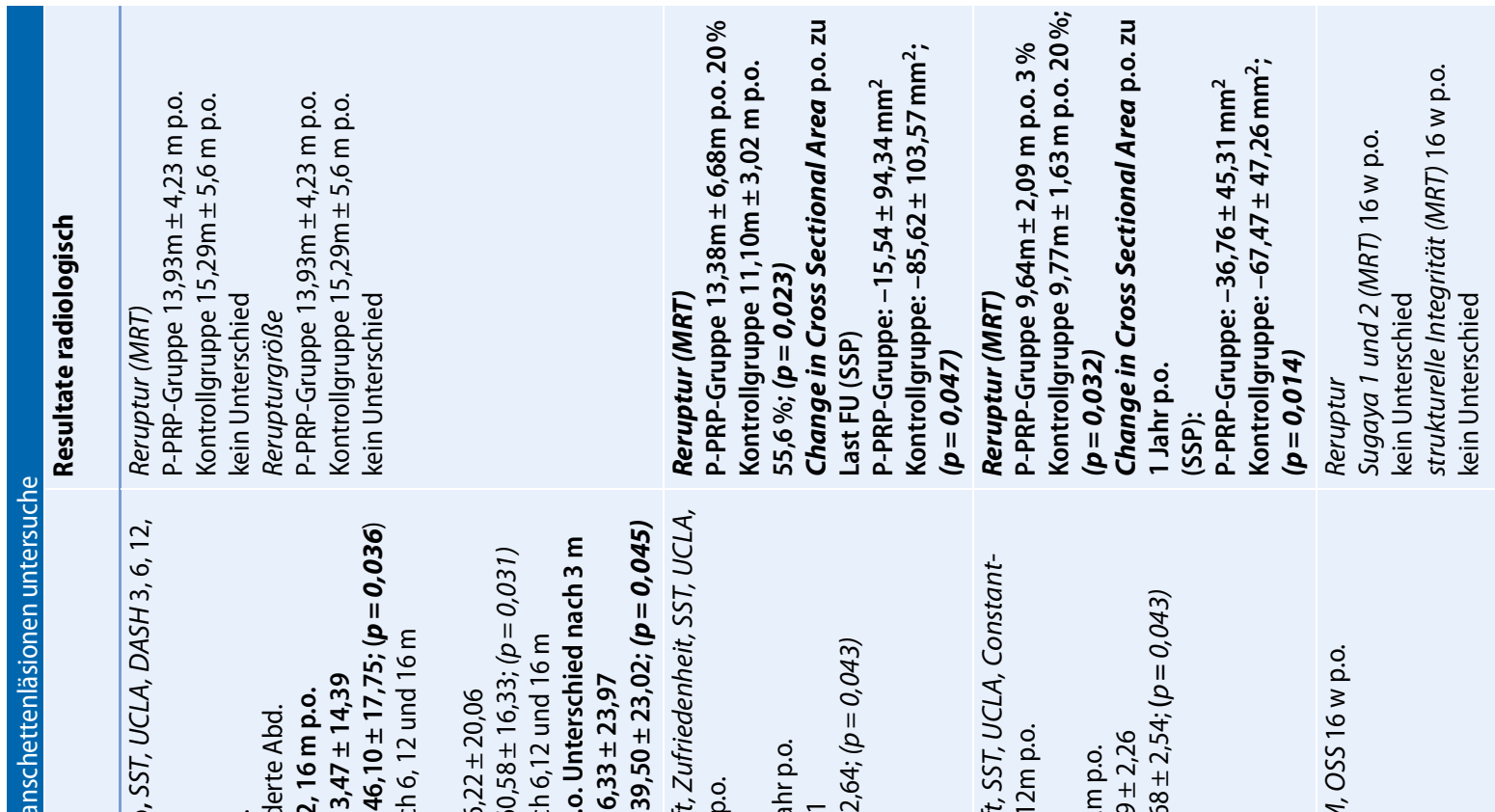

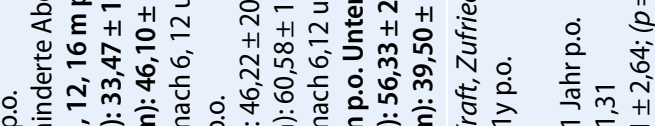

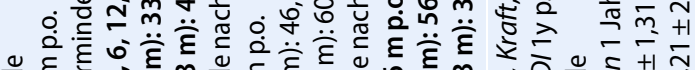

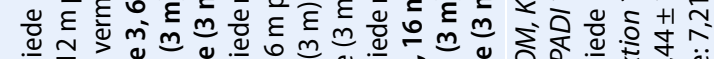

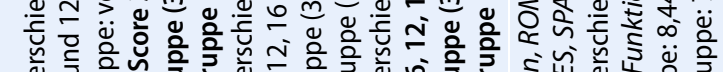

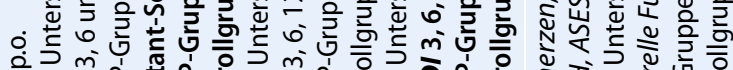
हैं

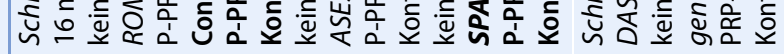

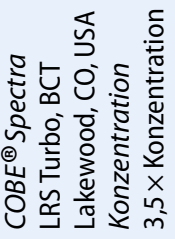

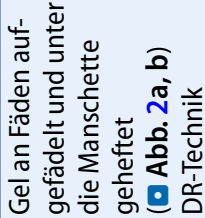

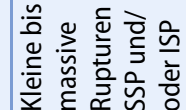
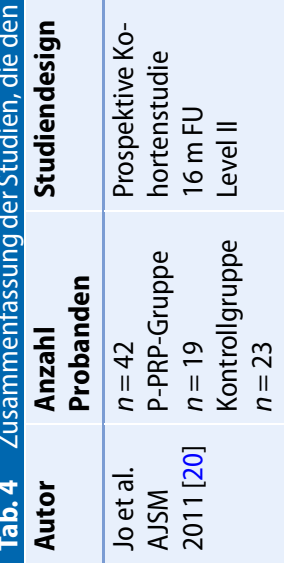
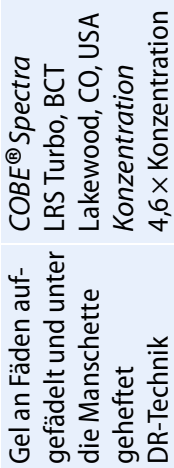

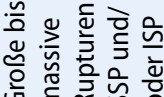
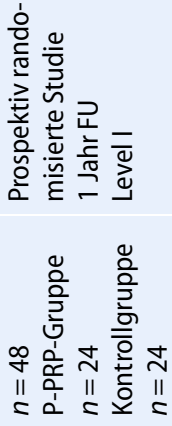

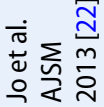

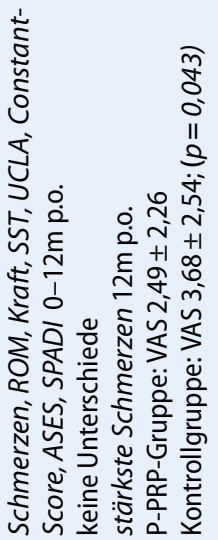

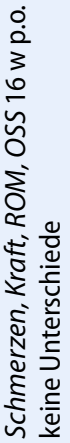
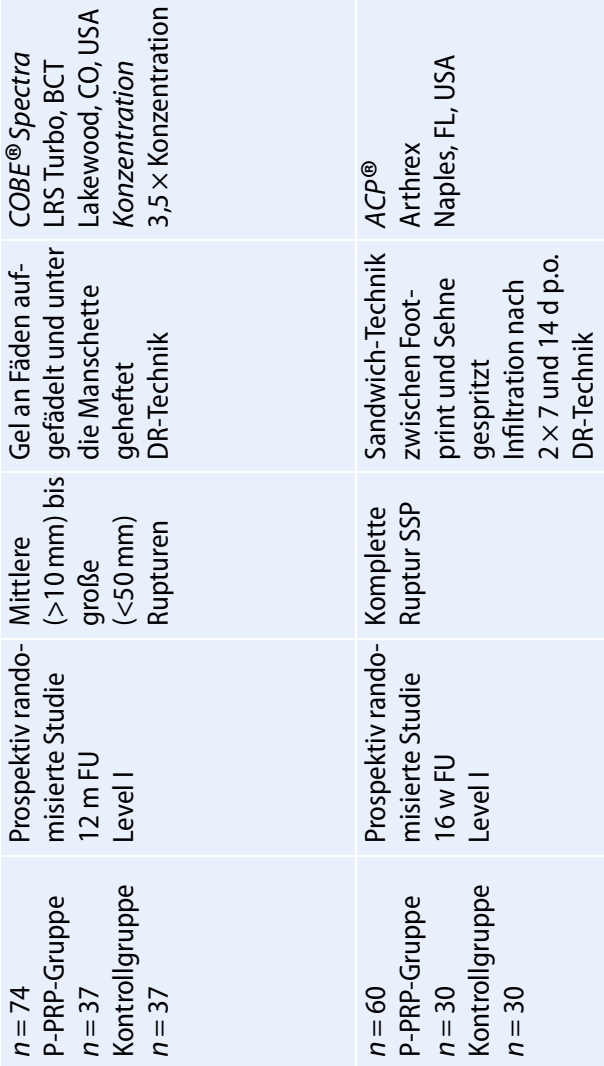

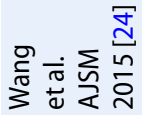




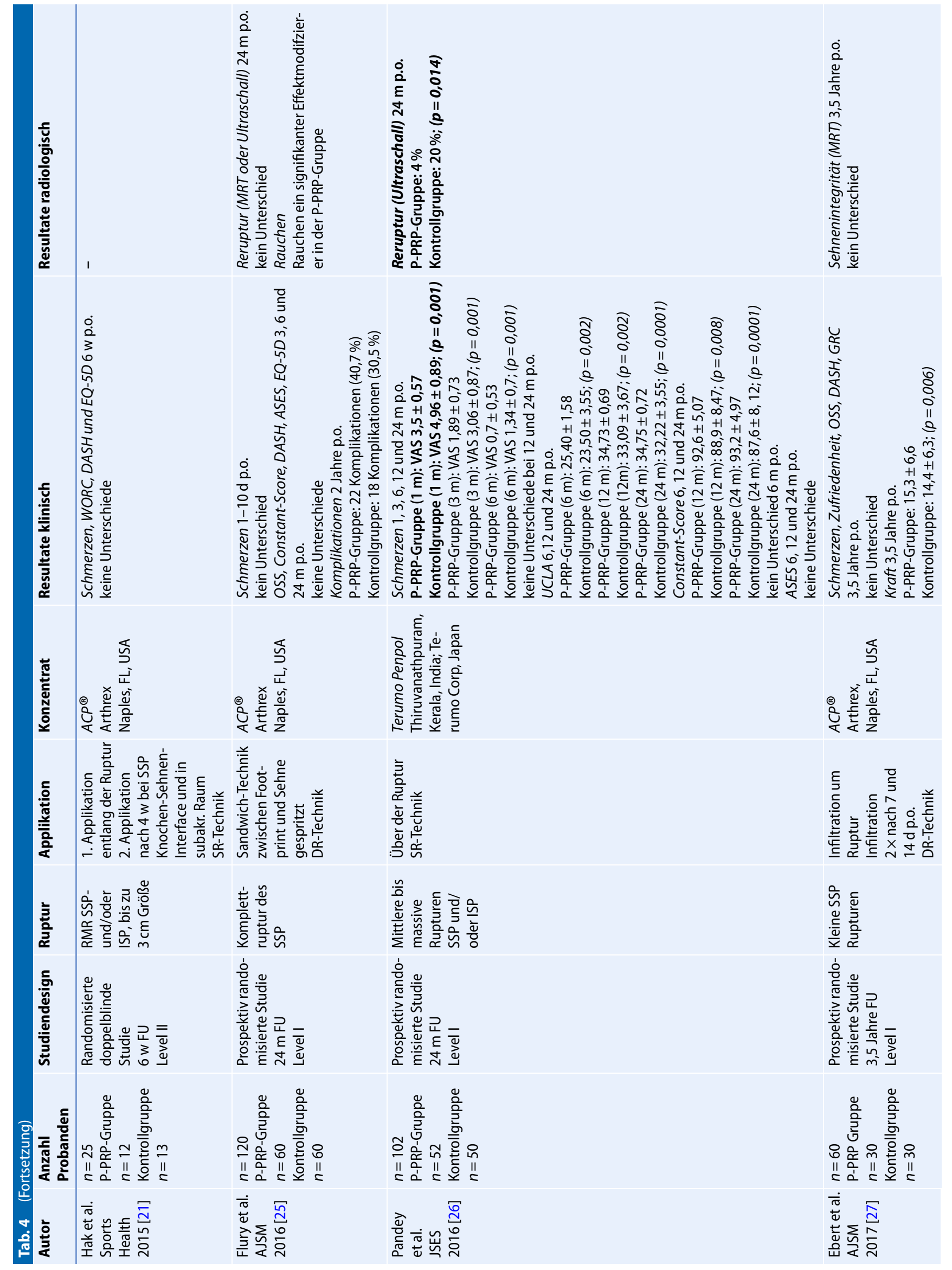




\section{Übersicht}

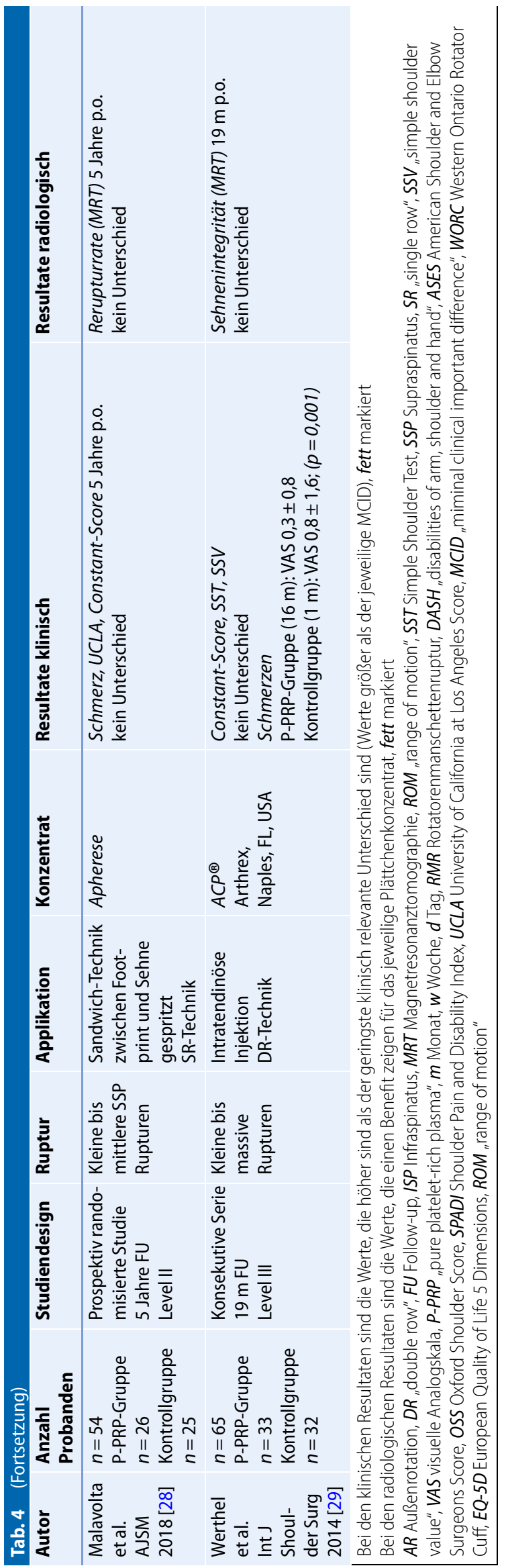

der P-PRF-Gruppe (56\%), die signifikant höher waren als in der Kontrollgruppe $(38 \%, p=0,024)$. P-PRF kann also zur biologischen Augmentation von RMRRefixationen nicht empfohlen werden.

\section{Nutzen der Plättchen- konzentrate für die RM-Heilung bei Partialrupturen}

Mehrere Studien zeigen ein verbessertes klinisches Outcome 3 Monate nach Plättchenkonzentratinfiltrationen bei partiellen RMR [44, 45]. Shams et al. [45] verglichen die Applikation von P-PRP mit einer Kortison-Infiltration in den Subakromialraum bei Patienten mit symptomatischer Partialruptur der RM. Im Gegensatz zu 12 Wochen p.o., wo sich signifikant bessere klinische Outcome-Scores zeigten, fanden sich 6 Monate p.o. keine Unterschiede zwischen den zwei Gruppen. Dies stellt den längerfristigen Nutzen einer P-PRP-Infiltration bei symptomatischen Partialrupturen in Frage.

Weitere Studien, in denen die Applikation von Plättchenkonzentraten entweder mit „dry needling“ [46], einer NaClInfiltration [47] oder Physiotherapie [48, 49] verglichen wurde, konnten keinen klaren Benefit der jeweiligen Plättchenkonzentrate im Vergleich zur jeweiligen Kontrolle zeigen. Carr et al. [50] applizierten L-PRP am Ende einer arthroskopischen Akromioplastik bei Patienten, die an einer symptomatischen RM-Tendinopathie litten. Auch hier zeigte sich kein Benefit der zusätzlichen L-PRP-Applikation.

Mit dieser limitieren Evidenz kann zum heutigen Zeitpunkt kein klares Statement bezüglich des Nutzens von Plättchenkonzentraten bei der konservativen Behandlung von Partialrupturen der RM abgegeben werden.

\section{Diskussion}

Bei der Evaluation des Nutzens von Plättchenkonzentraten ergibt sich mit der existierenden Evidenz kein klarer Benefit für die einzelnen Plättchenkonzentratuntergruppen. Einzig P-PRP (also pures leukozytenarmes PRP) kann empfohlen werden, denn in dieser Gruppe zeigt sich in 3 Level-I-Studien eine besse- 


\begin{tabular}{|c|c|c|c|c|c|}
\hline Outcome & $\begin{array}{l}\text { Anzahl } \\
\text { Patienten } \\
\text { P-PRP/ } \\
\text { Kontrolle }\end{array}$ & $\begin{array}{l}\text { Outcome } \\
\text { P-PRP/Kontrolle }\end{array}$ & Signifikanz & $\begin{array}{l}\text { Klinisch } \\
\text { relevanter } \\
\text { Unterschied } \\
\text { (>MCID) }\end{array}$ & $\begin{array}{l}\text { Gewinner } \\
\text { im } \\
\text { klinischen } \\
\text { Alltag }\end{array}$ \\
\hline \multicolumn{6}{|l|}{ P-PRP } \\
\hline Sehnenheilungsrate & $283 / 278$ & $17,0 \% / 30,9 \%$ & $P<0,05$ & - & P-PRP \\
\hline $\begin{array}{l}\text { Sehnenheilungsrate } \\
\text { in mittelgroßen } \\
\text { Rupturen }\end{array}$ & $105 / 98$ & $6,7 \% / 26,5 \%$ & $P<0,05$ & - & P-PRP \\
\hline $\begin{array}{l}\text { VAS-Score nach } 30 \\
\text { Tagen }\end{array}$ & $64 / 62$ & $3,3 / 4,9$ & $\mathrm{P}<0,05$ & JA & P-PRP \\
\hline $\begin{array}{l}\text { VAS-Score letzte } \\
\text { Untersuchung }\end{array}$ & 113/111 & $0,6 / 0,9$ & $P<0,05$ & NEIN & keiner \\
\hline Constant Score & $225 / 230$ & $87,1 / 84,3$ & $P<0,05$ & NEIN & keiner \\
\hline $\begin{array}{l}\text { University of } \\
\text { California, Los } \\
\text { Angeles Score }\end{array}$ & $172 / 169$ & $30,8 / 29,7$ & $P<0,05$ & NEIN & keiner \\
\hline $\begin{array}{l}\text { American Shoulder } \\
\text { and Elbow } \\
\text { Surgeons Score }\end{array}$ & $198 / 203$ & $88,6 / 87,0$ & $P=0,20$ & NEIN & keiner \\
\hline $\begin{array}{r}\mathrm{L}=\text { leukozytenreich, } \mathrm{P}= \\
\text { Analog Scaleauf einer } \mathrm{S} \\
\text { minimaler Unterschied } \\
\text { Roter Kasten = Klinisch } \\
\text { Untersch } \\
\text { Grüner Kasten = Für die } \\
\text { Refixat }\end{array}$ & $\begin{array}{l}\text { ozytenarm, } \\
\text { rzskala von } \\
\text { nte Resultat } \\
\text { (MCID VAS: } \\
\text { nten relevar } \\
\text { mittels P-PF }\end{array}$ & $\begin{array}{l}\text { = Plättchen-Reich } \\
\text {, MCID = Gerings } \\
\text { Resultat höher als } \\
\text { Benefit bei der AL }\end{array}$ & $\begin{array}{l}\text { Plasma, VAS } \\
\text { klinisch releve } \\
\text { iimale kliniscl } \\
\text { entation von }\end{array}$ & $\begin{array}{l}\text { Visual } \\
\text { ter } \\
\text { relevante } \\
\text { MRR }\end{array}$ & \\
\hline
\end{tabular}

Abb. 7 ム Metaanalyse von Hurley et al. aus dem Jahr 2019[13] aller Studien, bei denen der Benefit von P-PRP („pure platelet-rich plasma“) auf die Rotatorenmanschettenheilung untersucht wurde: grün Werte, bei denen für die Patienten in der Metaanalyse ein signifikanter Benefit besteht nach P-PRPAugmentation von Rotatorenmanschettenrekonstruktionen, rot der klinische Wert (Schmerzen 30 Tage p.o.), der über dem MCID für Schmerz von 1,4 liegt (RMR Rotatorenmanschettenruptur, Pleukozytenarm, PRP plättchenreiches Plasma, VAS visuellle Analogskala auf einer Schmerzskala von 0-10, RMR Rotatorenmanschettenruptur, $M C I D$ geringster klinisch relevanter minimalerUnterschied; roter Kasten klinisch relevante Resultate [Resultat höher als minimale klinisch relevante Unterschiede: MCIDVAS: 1,4]; grüner Kasten für die Patienten relevanter Benefit bei der Augmentation von RMR-Refixationen mittels P-PRP)

re Sehnenheilung. Dieses Resultat wird durch eine Metaanalyse gestützt, bei der Hurley et al. [13] über alle eingeschlossenen P-PRP-Studien nach Applikation von P-PRP eine verminderte Rerupturrate zeigen konnte. Klinisch zeigten sich zwar weniger Schmerzen 30 Tage und 16 Monate p.o. sowie vereinzelt bessere Outcome-Scores. Diese Unterschiede waren aber klinisch nicht relevant, was den Nutzen von P-PRP etwas relativiert. Auch bei der konservativen Behandlung von Partialrupturen zeigt sich ein heterogenes Resultat. Mit der limitierten Evidenz kann zum heutigen Zeitpunkt kein klares Statement bezüglich des Nutzens von Plättchenkonzentraten abgegeben werden.

Grundsätzlich wären dringend gute klinische prospektiv randomisierte Stu- dien nötig, die insbesondere den Nutzen von P-PRP (dem vielversprechendsten Kandidaten der vier Untergruppen) weiter untersuchen sollten.

\section{Fazit für die Praxis}

- Mit der heutigen Evidenz zeigen sich klare Unterschiede in den Ergebnissen für die verschiedenen Zusammensetzungen von Plättchenkonzentraten. Als einziges Produkt kann P-PRP („pure platelet-rich plasma") zur biologischen Augmentation von Rotatorenmanschettenrupturrefixationen empfohlen werden.

- In 3 verschiedenen Level-I-Studien zeigte sich eine signifikant tiefere Rerupturrate in den P-PRP-Gruppen bei Rupturen $>1 \mathrm{~cm}$. Aber auch in dieser Plättchenuntergruppe sind die Resultate heterogen: 6 andere Studien (3-mal Level-I-, 2-mal LevelII- und einmal Level-III-Studien) zeigten keine signifikanten Unterschiede bezüglich der Rerrupturrate.

- In der klinischen Untersuchung stehen die verminderten Schmerzen 30 Tage und 16 Monate postoperativ den fehlenden Unterschieden bei fast allen anderen klinischen OutcomeScores gegenüber.

- Erst nach Poolen der Daten im Rahmen einer Metaanalyse zeigt sich sowohl eine signifikant verbesserte Sehnenheilungsrate sowie weniger Schmerzen nach 30 Tagen. Weitere Studien sind hier erforderlich, um einen klaren Benefit von P-PRP aufzuzeigen.

- Bei der konservativen Therapie von Manschettenpartialrupturen und Tendinopathien zeigt sich kein Benefit.

\section{Korrespondenzadresse}

\section{Dr. Michael Schär}

Abteilung für Orthopädische Chirurgie und Traumatologie, Inselspital, Universität Bern Freiburgstrasse, 3010 Bern, Schweiz michael.schaer@insel.ch

Funding. Open access funding provided by University of Bern

\section{Einhaltung ethischer Richtlinien}

Interessenkonflikt. M. Zumstein erhält ein Beraterhonorar und einen Grant von der Firma Medacta. M. Schär erhält einen Grant von der Firma Medacta. R. Blunier und M.L. Frommer geben an, dass kein Interessenkonflikt besteht.

Für diesen Beitrag wurden von den Autoren keine Studien an Menschen oder Tieren durchgeführt. Für die aufgeführten Studien gelten die jeweils dort angegebenen ethischen Richtlinien.

Open Access. Dieser Artikel wird unter der Creative Commons Namensnennung 4.0 International Lizenz veröffentlicht, welche die Nutzung, Vervielfältigung, Bearbeitung, Verbreitung und Wiedergabe in jeglichem Medium und Format erlaubt, sofern Sie den/die ursprünglichen Autor(en) und die Quelle ordnungsgemäß nennen, einen Link zur Creative Commons Lizenz beifügen und angeben, ob Änderungen vorgenommen wurden.

Die in diesem Artikel enthaltenen Bilder und sonstiges Drittmaterial unterliegen ebenfalls der genannten 


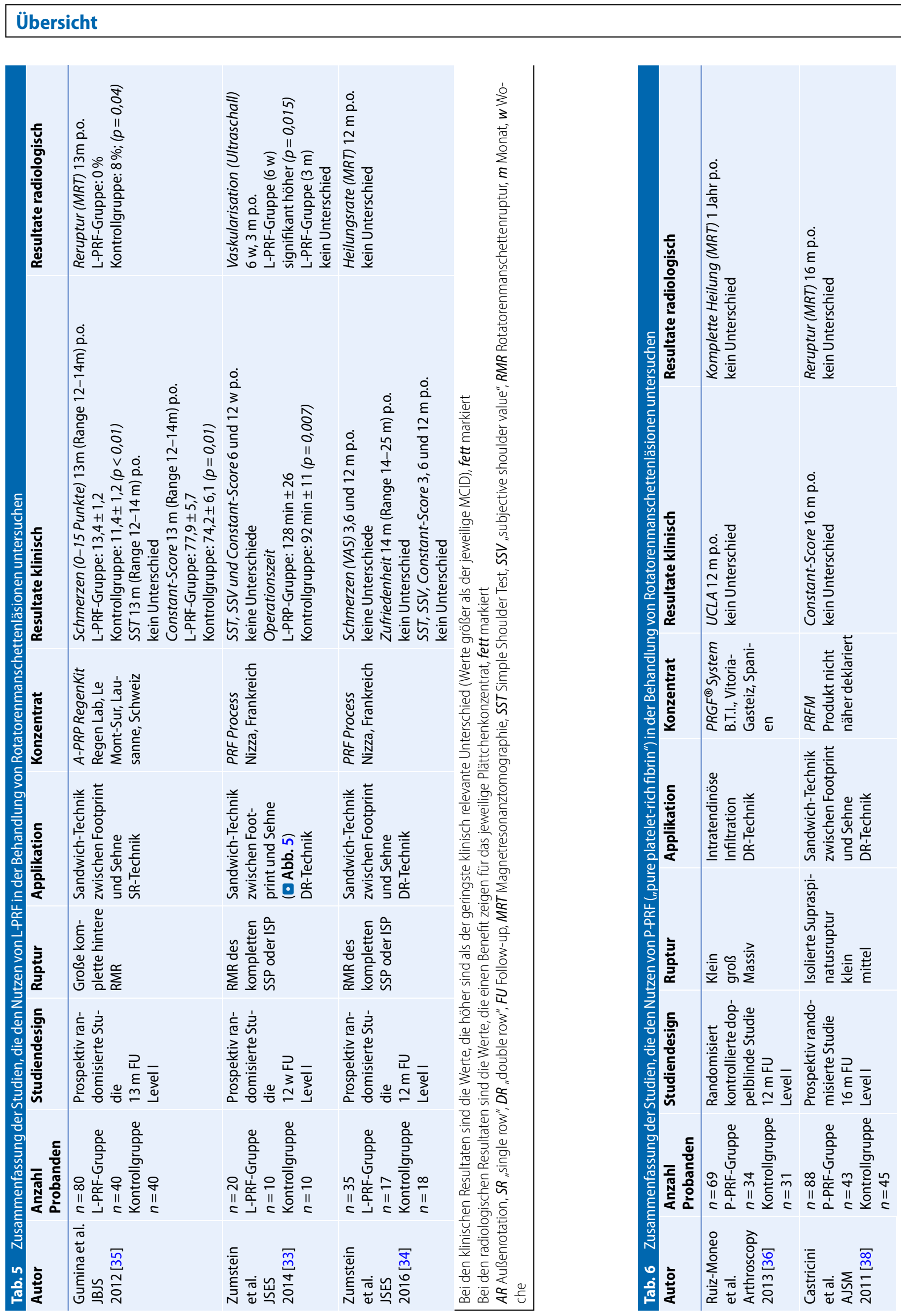




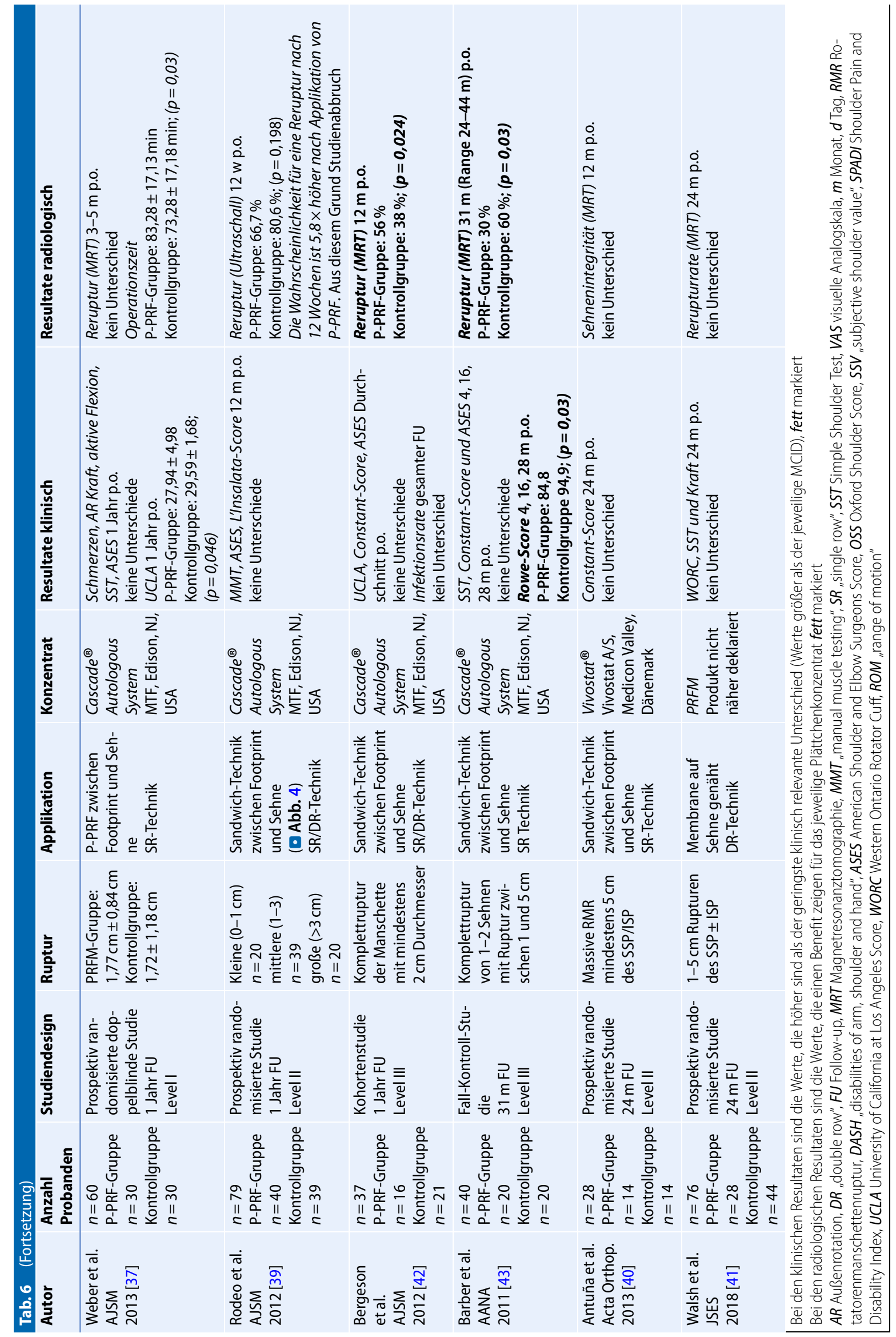




\begin{tabular}{|c|c|}
\hline \multicolumn{2}{|c|}{ Abkürzungen } \\
\hline$A R$ & Außenrotation \\
\hline ASES & $\begin{array}{l}\text { American Shoulder and Elbow } \\
\text { Surgeons Score }\end{array}$ \\
\hline DASH & $\begin{array}{l}\text { "Disabilities of arm, shoulder and } \\
\text { hand" }\end{array}$ \\
\hline$D R$ & „Double row" \\
\hline$E Q-5 D$ & $\begin{array}{l}\text { European Quality of Life } 5 \text { Dimen- } \\
\text { sions }\end{array}$ \\
\hline FU & Follow-up \\
\hline$I S P$ & Infraspinatus \\
\hline$L$ & Leukozytenreich \\
\hline$L-P R F$ & $\begin{array}{l}\text { "Leucocyte and platelet-rich } \\
\text { fibrin" }\end{array}$ \\
\hline$L-P R P$ & $\begin{array}{l}\text { "Leucocyte and platelet-rich } \\
\text { plasma" }\end{array}$ \\
\hline$M C I D$ & $\begin{array}{l}\text { "Miminal clinical important } \\
\text { difference" }\end{array}$ \\
\hline MRT & Magnetresonanztomographie \\
\hline OSS & Oxford Shoulder Score \\
\hline$P R P$ & „Platelet-rich plasma“ \\
\hline P-PRF & „Pure platelet-rich fibrin“ \\
\hline$P-P R P$ & „Pure platelet-rich plasma“ \\
\hline$R C T$ & „Randomized controlled trial" \\
\hline$R M$ & Rotatorenmanschette \\
\hline$R M R$ & Rotatorenmanschettenruptur \\
\hline ROM & „Range of motion" \\
\hline SPADI & Shoulder Pain and Disability Index \\
\hline$S R$ & "Single row" \\
\hline SSP & Supraspinatus \\
\hline SST & Simple Shoulder Test \\
\hline SSV & "Subjective shoulder value" \\
\hline$U C L A$ & $\begin{array}{l}\text { University of California at Los } \\
\text { Angeles Score }\end{array}$ \\
\hline VAS & Visuelle Analogskala \\
\hline WORC & Western Ontario Rotator Cuff \\
\hline
\end{tabular}

Creative Commons Lizenz, sofern sich aus der Abbildungslegende nichts anderes ergibt. Sofern das betreffende Material nicht unter der genannten Creative Commons Lizenz steht und die betreffende Handlung nicht nach gesetzlichen Vorschriften erlaubt ist, ist für die oben aufgeführten Weiterverwendungen des $\mathrm{Ma}$ terials die Einwilligung des jeweiligen Rechteinhabers einzuholen.
Weitere Details zur Lizenz entnehmen Sie bitte der Lizenzinformation auf http://creativecommons.org/ licenses/by/4.0/deed.de.

\section{Literatur}

1. Boileau P et al (2005) Arthroscopic repair of full-thickness tears of the supraspinatus: does the tendon really heal? J Bone Joint Surg Am 87(6):1229-1240

2. Franceschi $F$ et al (2007) Equivalent clinical results of arthroscopic single-row and double-row suture anchor repair for rotator cuff tears: a randomized controlled trial. Am J Sports Med 35(8):1254-1260

3. Galatz LM et al (2004) The outcome and repair integrity of completely arthroscopically repaired large and massive rotator cuff tears. J Bone Joint Surg Am 86(2):219-224

4. James R et al (2008) Tendon: biology, biomechanics, repair, growth factors, and evolving treatment options. J Hand Surg Am 33(1):102-112

5. Sugaya $\mathrm{H}$ et al (2005) Functional and structural outcome after arthroscopic full-thickness rotator cuff repair: single-row versus dual-row fixation. Arthroscopy 21(11):1307-1316

6. McElvany MD et al (2015) Rotator cuff repair: published evidence on factors associated with repair integrity and clinical outcome. Am J Sports Med 43(2):491-500

7. Baksh N et al (2013) Platelet-rich plasma in tendon models: a systematic review of basic science literature. Arthroscopy 29(3):596-607

8. Foster TE et al (2009) Platelet-rich plasma: from basic science to clinical applications. Am J Sports Med 37(11):2259-2272

9. Ide J et al (2009) The effect of a local application of fibroblast growth factor- 2 on tendon-to-bone remodeling in rats with acute injury and repair of the supraspinatus tendon. J Shoulder Elbow Surg 18(3):391-398

10. Rajabi H et al (2015) The healing effects of aquatic activities and allogenic injection of platelet-rich plasma (PRP) on injuries of Achilles tendon in experimental rat. World J Plast Surg 4(1):66-73

11. Yamaguchi $\mathrm{K}$ et al (2006) The demographic and morphological features of rotator cuff disease. A comparison of asymptomatic and symptomatic shoulders. JBone Joint Surg Am 88(8):1699-1704

12. Moraes VY et al (2014) Platelet-rich therapies for musculoskeletal soft tissue injuries. Cochrane Database Syst Rev 4:CD10071

13. Hurley ET et al (2019) The efficacy of plateletrich plasma and platelet-rich fibrin in arthroscopic rotator cuff repair: a meta-analysis of randomized controlled trials. Am J Sports Med 47(3):753-761

14. Dohan Ehrenfest DM, Rasmusson L, Albrektsson T (2009) Classification of platelet concentrates: from pure platelet-rich plasma (P-PRP) to leucocyteand platelet-rich fibrin (L-PRF). Trends Biotechnol 27(3):158-167

15. Holtby Ret al (2016) Impact of platelet-rich plasma on arthroscopic repair of small- to medium-sized rotator cuff tears: a randomized controlled trial. Orthop J Sports Med 4(9):2325967116665595

16. Randelli $P$ et al (2011) Platelet rich plasma in arthroscopic rotator cuff repair: a prospective RCT study, 2-year follow-up. J Shoulder Elbow Surg 20(4):518-528

17. Zhang Z, Wang Y, Sun J(2016) The effect of plateletrich plasma on arthroscopic double-row rotator cuff repair: a clinical study with 12-month followup. Acta Orthop Traumatol Turc 50(2):191-197
18. Charousset $C$ et al (2014) Does autologous leukocyte-platelet-rich plasma improve tendon healing in arthroscopic repair of large or massive rotator cuff tears? Arthroscopy 30(4):428-435

19. D'Ambrosi R et al (2016) Platelet-rich plasma supplementation in arthroscopic repair of fullthickness rotator cuff tears: a randomized clinical trial. Musculoskelet Surg 100(1):25-32

20. Jo $\mathrm{CH}$ et al (2011) Does platelet-rich plasma accelerate recovery after rotator cuff repair? A prospective cohort study. Am J Sports Med 39(10):2082-2090

21. Hak A et al (2015) A double-blinded placebo randomized controlled trial evaluating shortterm efficacy of platelet-rich plasma in reducing postoperative pain after arthroscopic rotator cuff repair: a pilot study. Sports Health 7(1):58-66

22. Jo CH et al (2013) Platelet-rich plasma for arthroscopic repair of large to massive rotator cuff tears: a randomized, single-blind, parallel-group trial. Am JSports Med 41(10):2240-2248

23. Jo CH et al (2015) Platelet-rich plasma for arthroscopic repair of medium to large rotator cuff tears: a randomized controlled trial. Am J Sports Med 43(9):2102-2110

24. Wang A et al (2015) Do postoperative plateletrich plasma injections accelerate early tendon healing and functional recovery after arthroscopic supraspinatus repair? A randomized controlled trial. Am J Sports Med 43(6):1430-1437

25. Flury M et al (2016) Does pure platelet-rich plasma affect postoperative clinical outcomes after arthroscopic rotator cuff repair? A randomized controlled trial. Am J Sports Med 44(8):2136-2146

26. Pandey $V$ et al (2016) Does application of moderately concentrated platelet-rich plasma improve clinical and structural outcome after arthroscopic repair of medium-sized to large rotator cuff tear? A randomized controlled trial. JShoulder Elbow Surg 25(8):1312-1322

27. Ebert JR et al (2017) A midterm evaluation of postoperative platelet-rich plasma injections on arthroscopic supraspinatus repair: a randomized controlled trial. Am JSports Med 45(13):2965-2974

28. Malavolta EA et al (2018) Clinical and structural evaluations of rotator cuff repair with and without added platelet-rich plasma at 5-year follow-up: a prospective randomized study. Am J Sports Med 46(13):3134-3141

29. Werthel JD et al (2014) Arthroscopic double row cuff repair with suture-bridging and autologous conditioned plasma injection: functional and structural results. Int JShoulder Surg 8(4):101-106

30. Xu S et al (2020) Minimal clinically important difference of oxford, constant, and UCLA shoulder score for arthroscopic rotator cuff repair. J Orthop 19:21-27

31. Tashjian RZ et al (2009) Minimal clinically important differences (MCID) and patient acceptable symptomatic state (PASS) for visual analog scales (VAS) measuring pain in patients treated for rotato cuff disease. JShoulder Elbow Surg 18(6):927-932

32. Tashjian RZetal(2010)Minimal clinicallyimportant differences in ASES and simple shoulder test scores after nonoperative treatment of rotator cuff disease. JBone Joint Surg Am 92(2):296-303

33. Zumstein MA et al (2014) Increased vascularization during early healing after biologic augmentation in repair of chronic rotator cuff tears using autologous leukocyte- and platelet-rich fibrin (LPRF): a prospective randomized controlled pilot trial. JShoulder Elbow Surg 23(1):3-12

34. Zumstein MA et al (2016) SECEC research grant 2008 II: use of platelet- and leucocyte-rich fibrin 
(L-PRF) does not affect late rotator cuff tendon healing: a prospective randomized controlled study.J Shoulder Elbow Surg 25(1):2-11

35. Gumina $S$ et al (2012) Use of platelet-leukocyte membrane in arthroscopic repair of large rotator cuff tears: a prospective randomized study. J Bone Joint Surg Am 94(15):1345-1352

36. Ruiz-Moneo P et al (2013) Plasma rich in growth factors in arthroscopic rotator cuff repair: a randomized, double-blind, controlled clinical trial. Arthroscopy 29(1):2-9

37. Weber SC et al (2013) Platelet-rich fibrin matrix in the management of arthroscopic repair of the rotator cuff: a prospective, randomized, doubleblinded study. Am J Sports Med 41 (2):263-270

38. Castricini R et al (2011) Platelet-rich plasma augmentation for arthroscopic rotator cuff repair: a randomized controlled trial. Am J Sports Med 39(2):258-265

39. Rodeo SA et al (2012) The effect of plateletrich fibrin matrix on rotator cuff tendon healing: a prospective, randomized clinical study. Am J Sports Med 40(6):1234-1241

40. Antuna $S$ et al (2013) Platelet-rich fibrin in arthroscopic repair of massive rotator cuff tears: a prospective randomized pilot clinical trial. Acta Orthop Belg 79(1):25-30

41. Walsh MR et al (2018) Platelet-rich plasma in fibrin matrix to augment rotator cuff repair: a prospective, single-blinded, randomized study with 2-year follow-up. J Shoulder Elbow Surg 27(9):1553-1563

42. Bergeson AG et al (2012) Effects of platelet-rich fibrin matrix on repair integrity of at-risk rotator cuff tears. Am J Sports Med 40(2):286-293

43. Barber FA et al (2011) Rotator cuff repair healing influenced by platelet-rich plasma construct augmentation. Arthroscopy 27(8):1029-1035

44. Sengodan VC, Kurian S, Ramasamy R (2017) Treatment of partial rotator cuff tear with ultrasound-guided platelet-rich plasma. J Clin Imaging Sci 7:32

45. Shams A et al (2016) Subacromial injection of autologous platelet-rich plasma versus corticosteroid for the treatment of symptomatic partia rotator cuff tears. Eur J Orthop Surg Traumatol 26(8):837-842

46. Rha DW et al (2013) Comparison of the therapeutic effects of ultrasound-guided platelet-rich plasma injection and dry needling in rotator cuff disease: a randomized controlled trial. Clin Rehabil 27(2):113-122

47. Kesikburun S et al (2013) Platelet-rich plasma injections in the treatment of chronic rotator cuff tendinopathy: a randomized controlled trial with 1-year follow-up. Am J Sports Med 41(11):2609-2616

48. Ilhanli I, Guder N, Gul M (2015) Platelet-rich plasma treatment with physical therapy in chronic partial supraspinatus tears. Iran Red Crescent Med J 17(9):e23732

49. Nejati $P$ et al (2017) Treatment of subacromial impingement syndrome: platelet-rich plasma or exercise therapy? A randomized controlled trial. Orthop J Sports Med 5(5):2325967117702366

50. Carr AJ et al (2015) Platelet-rich plasma injection with arthroscopicacromioplasty for chronic rotator cuff tendinopathy: a randomized controlled trial. Am JSports Med 43(12):2891-2897 